\title{
A HYBRID METHOD FOR INVERSE SCATTERING FOR SHAPE AND IMPEDANCE
}

\author{
Pedro Serranho \\ Institut für Numerische und Angewandte Mathematik, Georg-August Universität, \\ Lotzestrasse 16-18, D-37083 Göttingen, Germany \\ and \\ CeMat, Instituto Superior Técnico, Lisbon, Portugal \\ E-mail: serranho@math.uni-goettingen.de
}




\begin{abstract}
We present a hybrid method to numerically solve the inverse scattering problem for shape and impedance, given the far-field pattern for one incident direction. This method combines ideas of both iterative and decomposition methods, inheriting advantages of each of them, such as getting good reconstructions and not needing a forward solver at each step. An optimization problem is presented as the theoretic background of the method and numerical results show its feasibility.
\end{abstract}

AMS classification scheme numbers: 81Uxx

\title{
1. Introduction
}

Nondestructive obstacle detecting through low frequency wave propagation motivates a number of challenging mathematical and numerical problems with several applications such as radar and sonar or medical imaging. Among these problems, we are interested in numerical methods for reconstructing unaccessible impenetrable scattering obstacles and its unknown impedance value at the boundary within a homogeneous background from the knowledge of the incident field and the scattered field at large distances (far field pattern). We confine ourselves to the case of time harmonic acoustic waves.

Given an open bounded obstacle $D \subset \mathbb{R}^{2}$ with an unbounded and connected complement and an incident field $u^{i}$, the direct scattering problem consists of finding the total field $u=u^{i}+u^{s}$ as the sum of the known incident field $u^{i}$ and the scattered field $u^{s}$ such that both the Helmholtz equation

$$
\Delta u+k^{2} u=0 \quad \text { in } \mathbb{R}^{2} \backslash D
$$

with wave number $k>0$ and the impedance boundary condition

$$
\frac{\partial u}{\partial \nu}+i \lambda u=0 \quad \text { on } \Gamma:=\partial D
$$

are satisfied, where $\nu$ stands for the exterior normal vector to $\Gamma$ and the continuous realvalued function $\lambda \geq 0$ is the impedance and is considered to be known in the formulation of the direct problem. As particular cases of (2) one has the Neumann boundary condition which corresponds to $\lambda=0$ and the Dirichlet boundary condition which can be interpreted as $\lambda \rightarrow \infty$. The obstacle is then called sound-hard or sound-soft, respectively.

To ensure well-posedness, at infinity one needs to impose the Sommerfeld radiation condition

$$
\lim _{r \rightarrow \infty} \sqrt{r}\left(\frac{\partial u^{s}}{\partial r}-i k u^{s}\right)=0, \quad r=|x|
$$

with the limit satisfied uniformly in all directions. Then it is known (e.g. [4, Ch.2]) that the solution $u^{s}$ has an asymptotic behaviour of the form

$$
u^{s}(x)=\frac{e^{i k|x|}}{\sqrt{|x|}}\left(u_{\infty}(\hat{x})+O\left(\frac{1}{|x|}\right)\right), \quad|x| \rightarrow \infty,
$$

where $\hat{x}=x /|x|$. The function $u_{\infty}$ defined on the unit circle $\Omega$ is denoted as the far field pattern of $u^{s}$. By Rellich's lemma the scattered field $u^{s}$ is completely determined by its far field pattern. 
The inverse problem that we are interested in is to determine both the position and shape of the obstacle $D$ as well as the impedance $\lambda$, given the far-field pattern $u_{\infty}$ for only one incident wave. However, most of the methods developed to solve the inverse scattering problem only recover the position and the shape of the obstacle $D$. In general, to achieve that, iterative and decomposition methods (e.g. [19] and [9], respectively) require a priori knowledge of the impedance $\lambda$ while sampling methods (e.g. [3]) do not, though the latter requires much more data then just the far field pattern for one incident wave. Only recently methods to recover both the obstacle $D$ and the impedance $\lambda$ were developed, as the iterative method [14] and the method to recover the impedance in [1], applied after the obstacle was reconstruct by some sampling method.

In order to present the hybrid method proposed in this paper we will now focus on regularized iterative methods and decomposition methods. For a fixed incident field $u^{i}$, the solution to the direct scattering problem defines the operator

$$
F:(\gamma, \zeta) \mapsto u_{\infty}
$$

that maps the pair $(\gamma, \zeta)$ onto the far field corresponding to scattering by the obstacle with boundary $\gamma$ and impedance $\zeta$. In this sense, given the far field pattern $u_{\infty}$, the inverse problem is equivalent to finding the solution to the nonlinear and ill-posed operator equation

$$
F(\Gamma, \lambda)=u_{\infty}
$$

for both the unknown boundary $\Gamma$ and the unknown impedance $\lambda$. For the case where $\lambda$ is known, in particular for sound-soft or sound-hard obstacles, regularized Newton iterations applied to (5) have been studied and used for over two decades (see [7, 18, 19]). Their idea is to linearize (5), based on the Fréchet differentiability of the operator $F$ (see $[5,17]$ ) and iterate this procedure. Due to the ill-posedness of $F$ regularization is required in each iteration step. The main drawback of this method is that it requires the solution to the direct scattering problem at each iteration step and a reasonable initial guess to start the iterations.

On the other hand, decomposition methods take care of the ill-posedness and the nonlinearity of the inverse scattering problem separately. However, to our knowledge, methods of this class were only applied to recover the obstacle $D$ given a known impedance $\lambda$. Their idea is the following: In a first step the function $u$ is reconstructed from the given far field pattern $u_{\infty}$, for example, based on an analytic continuation principle, by representing the scattered field $u^{s}$ as a layer potential on an approximate boundary $\gamma$, usually considered to be inside $D$. The requirement that the far field of the potential coincides with the given far field $u_{\infty}$ leads to an ill-posed linear integral equation that can be approximately solved via Tikhonov regularization. Then in a second step one tries to find the boundary $\Gamma$ as the location where the boundary condition (2) is satisfied in a least squares sense. Though this method does not need the solution to the forward problem, the reconstructions obtained are not as accurate as those obtained by Newton iterations. There is also a gap between the theoretical background and the numerical implementation of the method.

In this work the idea is to develop a method combining ideas of both this two methods that not only recovers the obstacle $D$ but also the unknown impedance $\lambda$, requiring just one incident wave as in [14]. 
In [13] it was suggested combining ideas of both of these two reconstruction methods in order to create an iterative method using as background idea analytic continuation of the total field. In this sense this method is called hybrid. The same idea was applied to an inverse boundary value problem in potential theory [2] and to inverse scattering from soundsoft cracks [15] and from sound-hard obstacles [16]. This new method does not need a forward solver and the accuracy of the reconstructions is as satisfactory as for the Newton iterations, provided the initial guess is close enough to the exact boundary. In the present paper we describe an extension of the hybrid method to inverse scattering for shape and impedance.

This paper is organized as follows. In section 2 the direct scattering problem is solved by an integral equation method, in order to introduce layer potentials and other important concepts that we will need throughout the paper. In section 3 the operator $G$ is introduced as being the operator that for a given $C^{2}$-smooth field $u$ maps a curve $\gamma$ and a function $\zeta$ onto the boundary condition with impedance $\zeta$ of that field $u$ over $\gamma$. The Fréchet differentiability of $G$ is also settled. In section 4 the inverse scattering problem is posed in terms of $G$ and the hybrid method is proposed to numerically solve it. In section 5 an optimization problem is presented as the background for the method and finally, in section 6 , the numerical procedure is explained and some numerical reconstructions are presented to show the feasibility of the method.

\section{The Direct Problem}

To introduce notations, we briefly discuss the solution to the direct scattering problem via the combined single and double-layer potential approach. For details we refer, for example, to [4, Ch.3]. Given the domain $D$ with boundary $\Gamma$ of class $C^{2}$ and the incident field $u^{i}$, we want to find the uniquely determined scattered field $u^{s}$ such that (1)-(3) are satisfied. By

$$
\Phi(x, y)=\frac{i}{4} H_{0}^{(1)}(k|x-y|)
$$

we denote the fundamental solution to the two-dimensional Helmholtz equation in terms of the Hankel function $H_{0}^{(1)}$ of the first kind and order zero. After introducing the single and double-layer potential with density $\varphi$ on a closed $C^{2}$-curve $\gamma$ by

$$
\begin{aligned}
& \left(S_{\gamma} \varphi\right)(x):=\int_{\gamma} \Phi(x, y) \varphi(y) d s(y), \quad x \in \mathbb{R}^{2}, \\
& \left(K_{\gamma} \varphi\right)(x):=\int_{\gamma} \frac{\partial \Phi(x, y)}{\partial \nu(y)} \varphi(y) d s(y), \quad x \in \mathbb{R}^{2},
\end{aligned}
$$

respectively, we represent $u^{s}$ as a combined single and double-layer potential, that is, in the form

$$
u^{s}=\left(K_{\Gamma}-i \eta S_{\Gamma}\right) \varphi \quad \text { in } \mathbb{R}^{2} \backslash \bar{D}
$$

with some fixed coupling term $\eta>0$. Since $u^{s}$ given by (8) satisfies the Helmholtz equation and the Sommerfeld radiation condition $\varphi$ has to be determined such that the boundary condition (2) is satisfied. 
By the jump relations, considering the combined layer potential (8) as defined in $\mathbb{R}^{2} \backslash \Gamma$, its trace on $\Gamma$ is given by

$$
u_{ \pm}^{s}= \pm \frac{\varphi}{2}+\left(K_{\Gamma}-i \eta S_{\Gamma}\right) \varphi \quad \text { on } \Gamma,
$$

where \pm stands for the limit when approaching $\Gamma$ from outside and inside $D$, respectively. The normal trace of $u^{s}$ has also a jump and is given by

$$
\frac{\partial u^{s}}{\partial \nu}= \pm i \eta \frac{\varphi}{2}+\left(T_{\Gamma}-i \eta K_{\Gamma}^{*}\right) \varphi \quad \text { on } \Gamma
$$

in terms of $K_{\Gamma}^{*}$, which is the adjoint operator of $K_{\Gamma}$, and the hyper-singular operator

$$
\left(T_{\gamma} \varphi\right)(x):=\frac{\partial}{\partial \nu(x)} \int_{\gamma} \frac{\partial \Phi(x, y)}{\partial \nu(y)} \varphi(y) d s(y), \quad x \in \gamma
$$

In order to satisfy the boundary condition (2), the density $\varphi \in C^{1, \alpha}(\Gamma)$ has to be obtained as solution to

$$
B_{\Gamma, \lambda} \varphi=-\left(\frac{\partial u^{i}}{\partial \nu}+i \lambda u^{i}\right) \quad \text { on } \Gamma
$$

where

$$
B_{\gamma, \zeta}=i(\eta+\zeta) \frac{I}{2}+T_{\gamma}+i\left(\zeta K_{\gamma}-\eta K_{\gamma}^{*}\right)+\zeta \eta S_{\gamma}
$$

is the exterior trace operator $B_{\gamma, \zeta}: C^{1, \alpha}(\gamma) \rightarrow C^{0, \alpha}(\gamma)$ of the combined single and double layer potential and maps densities $\varphi$ in the Hölder space of uniformly continuously differentiable functions with exponent $\alpha>0$ into the space of Hölder continuous functions in $\gamma$.

Equation (10) is uniquely solvable (cf. [4]) and an approximate solution can be obtained, for example, by a collocation method as described in [12].

We note that via the asymptotics of the Hankel function the far field pattern of the combined layer potential (8) is given by

$$
u_{\infty}=F_{\infty, \Gamma} \varphi \quad \text { on } \Omega
$$

with $F_{\infty, \gamma}:=\left(K_{\infty, \gamma}-i \eta S_{\infty, \gamma}\right)$ given in terms of the far field operators

$$
\begin{aligned}
& \left(S_{\infty, \gamma} \varphi\right)(\hat{x})=\frac{e^{i \pi / 4}}{\sqrt{8 \pi k}} \int_{\gamma} e^{-i k \hat{x} \cdot y} \varphi(y) d s(y), \quad \hat{x} \in \Omega \\
& \left(K_{\infty, \gamma} \varphi\right)(\hat{x})=\frac{e^{i \pi / 4}}{\sqrt{8 \pi k}} \int_{\gamma} \frac{\partial e^{-i k \hat{x} \cdot y}}{\partial \nu(y)} \varphi(y) d s(y), \quad \hat{x} \in \Omega .
\end{aligned}
$$

\section{Differentiability with respect to the Boundary and the Impedance}

For the further analysis, a parameterization of the boundary curves is required. We assume that

$$
\gamma=\{z(s): s \in[0,2 \pi]\},
$$

with a $2 \pi$ periodic $C^{2}$-smooth function $z: \mathbb{R} \rightarrow \mathbb{R}^{2}$ and counter-clockwise orientation such that $\left.z\right|_{[0,2 \pi)}$ is injective. Our hybrid method is based on the linearization of the operator $G$ 
that, given a $C^{2}$-smooth field $u$ defined in a neighbourhood of $\gamma$, maps the pair $(z, \zeta)$, where $z$ parametrizes the contour $\gamma$ and $\zeta$ is an impedance function defined on $\gamma$, onto the impedance boundary condition trace of $u$ on $\gamma$, that is,

$$
G:\left.(z, \zeta) \mapsto(\nu \cdot \operatorname{grad} u+i \zeta u)\right|_{\gamma}
$$

or in a slight abuse of notation

$$
G:(z, \zeta) \mapsto \nu \cdot(\operatorname{grad} u \circ z)+i(\zeta u) \circ z \quad \text { in }[0,2 \pi] .
$$

In this section our task is to proof that the operator $G$ is Fréchet differentiable with respect to both the parameterization variable $z$ and the impedance variable $\zeta$. This will be of major importance for the presentation of the hybrid method in the next section. Clearly, the Fréchet differentiability of $G$ is related with the smoothness of both $u$ and $\zeta$. Considering $u$ to be $C^{2}$ smooth and assuming $\zeta$ to be $C^{1}$-smooth then $G$ is Fréchet differentiable with respect to both of this variables. Taking these assumptions our task is now just to characterize the Fréchet derivative $G^{\prime}$.

Remark 1 Later on in sections 4 and 6 the operator $G$ will be applied to the total field $u$ and the impedance $\lambda$ defined as in the previous section. Therefore the assumption on uholds true in applications since the total field $u$ is an analytic function in its domain of definition. However, for the scattering problem the impedance $\lambda$ is usually just assumed to be continuous on the boundary $\Gamma$. Though one needs that $\lambda$ is $C^{1}$-smooth for the following analysis, this problem can be overcome by a restriction to star-shaped domains, as we will see further on.

To simplify this task we will split the operator $G$ in a sum of two operators

$$
G(z, \zeta)=G_{1}(z)+i G_{2}(z, \zeta)
$$

We define

$$
G_{1}: z \mapsto \frac{z^{\prime \perp}}{\left|z^{\prime}\right|} \cdot(\operatorname{grad} u \circ z) \quad \text { in }[0,2 \pi]
$$

where $z^{\perp}=\left(z_{2},-z_{1}\right)$ and

$$
G_{2}:(z, \zeta) \mapsto(\zeta u) \circ z \quad \text { in }[0,2 \pi] .
$$

We note that the differentiability of $G_{1}$ was already considered in [16]. We formulate the result and revisit the proof after introducing some notation. We denote by $\tau$ the tangential vector to the boundary in the counter-clockwise direction, by $H$ the mean curvature and by $h_{\nu}, h_{\tau}$ the normal and tangential components of the vector $h$, respectively. We also note that

$$
h_{\tau}^{\prime}:=h^{\prime} \cdot \tau \quad \text { and } \quad h_{\nu}^{\prime}:=h^{\prime} \cdot \nu,
$$

and as $G_{1}$ only depends on $z$ we will denote for simplicity $G_{1}^{\prime}:=\partial G_{1} / \partial z$.

Theorem 1 The operator $G_{1}: C^{2}[0,2 \pi] \rightarrow C[0,2 \pi]$ is Fréchet differentiable and its derivative is given by

$$
G_{1}^{\prime}(z) h=-\frac{h_{\nu}^{\prime}}{\left|z^{\prime}\right|} \frac{\partial u}{\partial \tau} \circ z+\left[\frac{\partial^{2} u}{\partial \tau \partial \nu} \circ z-H \frac{\partial u}{\partial \tau} \circ z\right] h_{\tau}+h_{\nu} \frac{\partial^{2} u}{\partial \nu^{2}} \circ z
$$

in $[0,2 \pi]$. 
Proof. Let $h$ be sufficiently small to ensure that

$$
\gamma_{z+h}=\{z(s)+h(s): s \in[0,2 \pi]\}
$$

describes a closed curve.

We decompose

$$
\begin{aligned}
G_{1}(z+h)-G_{1}(z)= & \left(\frac{z^{\prime \perp}+h^{\prime \perp}}{\left|z^{\prime}+h^{\prime}\right|}-\frac{z^{\prime \perp}}{\left|z^{\prime}\right|}\right) \cdot(\operatorname{grad} u \circ(z+h)) \\
& +\frac{z^{\prime \perp}}{\left|z^{\prime}\right|} \cdot(\operatorname{grad} u \circ(z+h)-\operatorname{grad} u \circ z)
\end{aligned}
$$

and treat both terms on the right hand side separately. Using Taylor's formula, we begin by noting that

$$
\begin{aligned}
\frac{z^{\prime \perp}+h^{\prime \perp}}{\left|z^{\prime}+h^{\prime}\right|}-\frac{z^{\prime \perp}}{\left|z^{\prime}\right|} & =\frac{h^{\prime \perp}}{\left|z^{\prime}\right|}-\frac{z^{\prime \perp}\left(z^{\prime} \cdot h^{\prime}\right)}{\left|z^{\prime}\right|^{3}}+O\left(\left|h^{\prime}\right|^{2}\right) \\
& =\frac{1}{\left|z^{\prime}\right|}\left(h^{\prime \perp}-h_{\tau}^{\prime} \nu\right)+O\left(\left|h^{\prime}\right|^{2}\right)
\end{aligned}
$$

since $\tau=z^{\prime} /\left|z^{\prime}\right|$. Using

$$
\operatorname{grad} u \circ(z+h)-\operatorname{grad} u \circ z=O(|h|)
$$

we consequently have

$$
\begin{aligned}
& \left(\frac{z^{\prime \perp}+h^{\prime \perp}}{\left|z^{\prime}+h^{\prime}\right|}-\frac{z^{\prime \perp}}{\left|z^{\prime}\right|}\right) \cdot(\operatorname{grad} u \circ(z+h)) \\
& =\frac{1}{\left|z^{\prime}\right|}\left(h^{\perp}-h_{\tau}^{\prime} \nu\right) \cdot \operatorname{grad} u \circ z+O\left(\left|h^{\prime}\right|^{2}\right)+O\left(h^{\prime} \cdot h\right) .
\end{aligned}
$$

We now perform a change of variables in a neighbourhood of $\gamma$ by

$$
x(s, \varepsilon)=z(s)+\varepsilon \nu(s), \quad s \in[0,2 \pi], \varepsilon \in\left(-\varepsilon_{0}, \varepsilon_{0}\right)
$$

and set

$$
v(s, \varepsilon)=u(z(s)+\varepsilon \nu(s)) .
$$

In the new coordinate system we have that

$$
\operatorname{grad} v(s, \varepsilon)=\frac{1}{\left|z^{\prime}(s)+\varepsilon \nu^{\prime}(s)\right|^{2}} \frac{\partial v}{\partial s}(s, \varepsilon)\left[z^{\prime}(s)+\varepsilon \nu^{\prime}(s)\right]+\frac{\partial v}{\partial \varepsilon}(s, \varepsilon) \nu(s) .
$$

Therefore we can rewrite (19) as

$$
\begin{aligned}
\left(\frac{z^{\prime \perp}+h^{\prime \perp}}{\left|z^{\prime}+h^{\prime}\right|}-\frac{z^{\prime \perp}}{\left|z^{\prime}\right|}\right) \cdot(\operatorname{grad} u \circ(z+h))= & -\frac{h_{\nu}^{\prime}}{\left|z^{\prime}\right|^{2}} \frac{\partial v}{\partial s}+O\left(\left|h^{\prime}\right|^{2}\right) \\
& +O\left(\left|h^{\prime} \cdot h\right|\right)
\end{aligned}
$$

using the equalities

$$
h^{\prime \perp} \cdot \tau=-h_{\nu}^{\prime} \quad \text { and } \quad h^{\prime \perp} \cdot \nu=h_{\tau}^{\prime} .
$$


We now consider the second term on the right hand side of (18). Taylor's formula and the relations $\nu \cdot z^{\prime}=0$ and $\nu^{\prime} \cdot \nu=0$ imply that

$$
\begin{aligned}
\nu(s) & \cdot[\operatorname{grad} v(s+\sigma, \epsilon)-\operatorname{grad} v(s, 0)] \\
= & {\left[\frac{\partial^{2} v}{\partial s \partial \varepsilon}(s, 0)-H(s) \frac{\partial v}{\partial s}(s, 0)\right] \sigma+\frac{\partial^{2} v}{\partial \varepsilon^{2}}(s, 0) \epsilon+O\left(\sigma^{2}+\epsilon^{2}\right) }
\end{aligned}
$$

where the mean curvature $H$ in two dimensions is given by

$$
H=\frac{z_{1}^{\prime} z_{2}^{\prime \prime}-z_{2}^{\prime} z_{1}^{\prime \prime}}{\left|z^{\prime}\right|^{3}}=-\frac{z^{\prime \prime} \cdot \nu}{\left|z^{\prime}\right|^{2}}
$$

In view of the second term on the right hand side of (18) we want to choose the pair $(\sigma, \epsilon)$ such that

$$
z(s)+h(s)=z(s+\sigma)+\epsilon \nu(s+\sigma) .
$$

By Taylor's formula, we note that

$$
h(s)-\epsilon \nu(s)+O(\sigma \epsilon)=z(s+\sigma)-z(s)=z^{\prime}(s) \sigma+O\left(\sigma^{2}\right)
$$

and therefore

$$
h(s)=z^{\prime}(s) \sigma+\epsilon \nu(s)+O(\sigma \epsilon)+O\left(\sigma^{2}\right) .
$$

Comparing the previous expression with the decomposition

$$
h(s)=\frac{h_{\tau}(s)}{\left|z^{\prime}(s)\right|} z^{\prime}(s)+h_{\nu}(s) \nu(s),
$$

we have

$$
\sigma=\frac{h_{\tau}(s)}{\left|z^{\prime}(s)\right|} \quad \text { and } \quad \epsilon=h_{\nu}(s)
$$

Therefore, we can write the second term on the right hand side of (18) as

$$
\begin{aligned}
\nu(s) \cdot & (\operatorname{grad} u(z(s)+h(s))-\operatorname{grad} u(z(s)))= \\
& {\left[\frac{\partial^{2} v}{\partial s \partial \varepsilon}(s, 0)-H(s) \frac{\partial v}{\partial s}(s, 0)\right] \frac{h_{\tau}(s)}{\left|z^{\prime}(s)\right|}+\frac{\partial^{2} v}{\partial \varepsilon^{2}}(s, 0) h_{\nu}(s)+O\left(|h|^{2}\right) . }
\end{aligned}
$$

Inserting (21) and (23) into (18) and by definition of the Fréchet derivative

$$
\left|G_{1}(z+h)-G_{1}(z)-G_{1}^{\prime}(z) h\right|=O\left(\|h\|_{C^{2}}^{2}\right), \quad\|h\|_{C^{2}} \rightarrow 0
$$

one gets

$$
\begin{aligned}
G_{1}^{\prime}(z(s)) h(s)= & -\frac{h_{\nu}^{\prime}}{\left|z^{\prime}\right|^{2}} \frac{\partial v}{\partial s}(s, 0)+\left[\frac{\partial^{2} v}{\partial s \partial \varepsilon}(s, 0)-H(s) \frac{\partial v}{\partial s}(s, 0)\right] \frac{h_{\tau}(s)}{\left|z^{\prime}(s)\right|} \\
& +\frac{\partial^{2} v}{\partial \varepsilon^{2}}(s, 0) h_{\nu}(s)
\end{aligned}
$$

and by the relations

$$
\frac{\partial v}{\partial \varepsilon}(s, 0)=\frac{\partial u}{\partial \nu}(z(s))
$$


and

$$
\frac{1}{\left|z^{\prime}(s)\right|} \frac{\partial v}{\partial s}(s, 0)=\frac{\partial u}{\partial \tau}(z(s))
$$

the result follows.

In practice one wants to avoid computing the term $\partial^{2} u / \partial^{2} \nu$ appearing in (17). Therefore, in the following corollary this term is eliminated by using the fact that $u$ satisfies the Helmholtz equation.

Corollary 1 Provided u satisfies the Helmholtz equation, the Fréchet derivative of $G_{1}: C^{2}[0,2 \pi] \rightarrow C[0,2 \pi]$ is given by

$$
G_{1}^{\prime}(z) h=-k^{2} h_{\nu} u \circ z-\frac{\partial}{\partial \tau}\left(h_{\nu}\left(\frac{\partial u}{\partial \tau} \circ z\right)\right)+H h_{\nu} \frac{\partial u}{\partial \nu} \circ z+h_{\tau} \frac{\partial^{2} u}{\partial \tau \partial \nu} \circ z(27
$$

in $[0,2 \pi]$.

Proof. Using the same change of variables (20) as in the previous proof for the Laplace operator we have that

$$
\begin{aligned}
\Delta v(s, \varepsilon)= & \frac{1}{\left|z^{\prime}(s)+\varepsilon \nu^{\prime}(s)\right|}\left\{\frac{\partial}{\partial s}\left(\frac{1}{\left|z^{\prime}(s)+\varepsilon \nu^{\prime}(s)\right|} \frac{\partial v}{\partial s}(s, \varepsilon)\right)\right. \\
& \left.+\frac{\partial}{\partial \varepsilon}\left(\left|z^{\prime}(s)+\varepsilon \nu^{\prime}(s)\right| \frac{\partial v}{\partial \varepsilon}(s, \varepsilon)\right)\right\} .
\end{aligned}
$$

Therefore we can write

$$
\begin{aligned}
\frac{\partial^{2} v}{\partial \varepsilon^{2}}(s, 0)= & -k^{2} v(s, 0)+\frac{z^{\prime}(s) \cdot z^{\prime \prime}(s)}{\left|z^{\prime}(s)\right|^{4}} \frac{\partial v}{\partial s}(s, 0) \\
& -\frac{1}{\left|z^{\prime}(s)\right|^{2}} \frac{\partial^{2} v}{\partial s^{2}}(s, 0)+H(s) \frac{\partial v}{\partial \varepsilon}(s, 0)
\end{aligned}
$$

since $u$ satisfies the Helmholtz equation. This comes from the fact that in the new coordinate system

$$
\begin{aligned}
\Delta v(s, \epsilon) \rightarrow & -\frac{z^{\prime}(s) \cdot z^{\prime \prime}(s)}{\left|z^{\prime}(s)\right|^{4}} \frac{\partial v}{\partial s}(s, 0)+\frac{1}{\left|z^{\prime}(s)\right|^{2}} \frac{\partial^{2} v}{\partial s^{2}}(s, 0) \\
& +\frac{z^{\prime}(s) \cdot \nu^{\prime}(s)}{\left|z^{\prime}(s)\right|^{2}} \frac{\partial v}{\partial \varepsilon}(s, 0)+\frac{\partial^{2} v}{\partial \varepsilon^{2}}(s, 0),
\end{aligned}
$$

as $\epsilon \rightarrow 0$ and from using the identity

$$
\nu^{\prime} \cdot z^{\prime}=z^{\prime \prime} \cdot \nu=-\left|z^{\prime}\right|^{2} H .
$$

Substituting (28) in (24) one gets the characterization

$$
\begin{aligned}
G_{1}^{\prime}(z) h= & -\frac{h_{\nu}^{\prime}}{\left|z^{\prime}\right|^{2}} \frac{\partial v}{\partial s}+\left[\frac{\partial^{2} v}{\partial s \partial \varepsilon}-H \frac{\partial v}{\partial s}\right] \frac{h_{\tau}}{\left|z^{\prime}\right|} \\
& +\left[-k^{2} v+\frac{z^{\prime} \cdot z^{\prime \prime}}{\left|z^{\prime}\right|^{4}} \frac{\partial v}{\partial s}-\frac{1}{\left|z^{\prime}\right|^{2}} \frac{\partial^{2} v}{\partial s^{2}}+H \frac{\partial v}{\partial \varepsilon}\right] h_{\nu}
\end{aligned}
$$


or rearranging the terms

$$
\begin{aligned}
G_{1}^{\prime}(z)= & -k^{2} h_{\nu} v+H h_{\nu} \frac{\partial v}{\partial \varepsilon}+\frac{h_{\tau}}{\left|z^{\prime}\right|} \frac{\partial^{2} v}{\partial s \partial \varepsilon} \\
& -\frac{1}{\left|z^{\prime}\right|^{2}}\left[h_{\nu}^{\prime}-h_{\nu} \frac{z^{\prime} \cdot z^{\prime \prime}}{\left|z^{\prime}\right|^{2}}+h_{\tau} H\left|z^{\prime}\right|\right] \frac{\partial v}{\partial s}-\frac{h_{\nu}}{\left|z^{\prime}\right|^{2}} \frac{\partial^{2} v}{\partial s^{2}}
\end{aligned}
$$

where for simplicity $v$ holds for $v(s, 0)$.

Considering (26) one gets

$$
\frac{\partial}{\partial \tau}\left(h_{\nu}\left(\frac{\partial u}{\partial \tau} \circ z\right)\right)=\frac{1}{\left|z^{\prime}\right|^{2}}\left[h_{\nu}^{\prime}+h \cdot \nu^{\prime}-h_{\nu} \frac{z^{\prime} \cdot z^{\prime \prime}}{\left|z^{\prime}\right|^{2}}\right] \frac{\partial v}{\partial \nu}+\frac{h_{\nu}}{\left|z^{\prime}\right|^{2}} \frac{\partial^{2} v}{\partial s^{2}}
$$

and if one has the identity

$$
h \cdot \nu^{\prime}=-\frac{h_{\tau}}{\left|z^{\prime}\right|} \nu \cdot z^{\prime \prime}=h_{\tau} H\left|z^{\prime}\right|,
$$

one can substitute (31) in (30) and the latter in (29), obtaining the result by (25) and (26).

To prove (31) one starts by noting that

$$
h \cdot \nu^{\prime}=h \cdot\left(\frac{z^{\prime \prime}}{\left|z^{\prime}\right|}-\frac{z^{\prime \prime} \cdot z^{\prime}}{\left|z^{\prime}\right|^{2}} \nu\right)=-\frac{h^{\perp} \cdot z^{\prime \prime}}{\left|z^{\prime}\right|}-h_{\nu} \frac{z^{\prime \prime} \cdot \tau}{\left|z^{\prime}\right|} .
$$

Now one only needs to decompose $h$ in its tangential and normal component in order to get

$$
h^{\perp}=h_{\tau} \nu-h_{\nu} \tau
$$

and apply it in (32).

We will now characterize the Fréchet derivative of the operator $G_{2}$ defined in (16) on both the parameterization $z$ and on the impedance $\zeta$. For the derivative on the first variable we have the following result.

Theorem 2 The Fréchet derivative of $G_{2}: C^{2}[0,2 \pi] \times C^{1}\left(\mathbb{R}^{2}\right) \rightarrow C[0,2 \pi]$ with respect to the parameterization is given by

$$
\frac{\partial}{\partial z} G_{2}(z, \zeta) h=[(\zeta \operatorname{grad} u+u \operatorname{grad} \zeta) \circ z] \cdot h .
$$

Proof. We proceed as in the proof of Theorem 1. We first decompose

$$
\begin{aligned}
G_{2}(z+h, \zeta)-G_{2}(z, \zeta)= & (\zeta \circ(z+h)-\zeta \circ z)(u \circ(z+h)) \\
& +(\zeta \circ z)(u \circ(z+h)-u \circ z)
\end{aligned}
$$

and using Taylor's Formula

$$
\begin{aligned}
& u((z+h)(s))=u(z(s))+\operatorname{grad} u(z(s)) \cdot h(s)+O\left(|h|^{2}\right), \quad s \in[0,2 \pi] \\
& \zeta((z+h)(s))=\zeta(z(s))+\operatorname{grad} \zeta(z(s)) \cdot h(s)+O\left(|h|^{2}\right), \quad s \in[0,2 \pi]
\end{aligned}
$$

the result follows. 
As the operator $G_{2}$ is linear in the second variable, the Fréchet derivative with respect to the impedance is just given by

$$
\frac{\partial}{\partial \zeta} G_{2}(z, \zeta) \mu=(\mu u) \circ z
$$

Now combining (27),(33) and (34) we arrive at the following theorem.

Theorem 3 The Fréchet derivative of $G: C^{2}[0,2 \pi] \times C^{1}\left(\mathbb{R}^{2}\right) \rightarrow C[0,2 \pi]$ with respect to the parameterization is given by

$$
\begin{aligned}
\frac{\partial}{\partial z} G(z, \zeta) h= & -k^{2} h_{\nu} u \circ z-\frac{\partial}{\partial \tau}\left(h_{\nu}\left(\frac{\partial u}{\partial \tau} \circ z\right)\right)+(i \zeta+H) h_{\nu} \frac{\partial u}{\partial \nu} \circ z \\
& +h_{\tau}\left[\left(\frac{\partial^{2} u}{\partial \tau \partial \nu}+i \zeta \frac{\partial u}{\partial \tau}\right) \circ z\right]+i[(u \operatorname{grad} \lambda) \circ z] \cdot h
\end{aligned}
$$

and with respect to the impedance is given by

$$
\frac{\partial}{\partial \zeta} G(z, \zeta) \mu=(\mu u) \circ z
$$

both in $[0,2 \pi]$

As we will see in the next section, this last theorem is of crucial importance for the idea and implementation of the hybrid method. Remark 2 will show that this method requires the computation of more terms than the Newton method applied to the operator defined in (4). However, no forward problem must be solved throughout the process of computation of the derivative of $G$, whereas for computing $F^{\prime}$ this must be done.

Remark 2 One can also rewrite (35) in the following form

$$
\begin{aligned}
\frac{\partial}{\partial z} G(z, \zeta) h= & -k^{2} h_{\nu} u \circ z-\frac{\partial}{\partial \tau}\left(h_{\nu}\left(\frac{\partial u}{\partial \tau} \circ z\right)\right)+(i \zeta+H) h_{\nu} \frac{\partial u}{\partial \nu} \circ z \\
& +h_{\tau}\left(\frac{\partial}{\partial \tau}\left(\frac{\partial u}{\partial \nu}+i \zeta u\right) \circ z\right)+i h_{\nu}\left(\frac{\partial \zeta}{\partial \nu} u\right) \circ z
\end{aligned}
$$

since we have the decomposition in the normal and tangential direction

$$
h \cdot(\operatorname{grad} \zeta \circ z)=h_{\tau} \frac{\partial \zeta}{\partial \tau} \circ z+h_{\nu} \frac{\partial \zeta}{\partial \nu} \circ z
$$

Note that if $z$ is the parameterization to the true boundary solution $\Gamma$ and $\zeta$ is the true impedance solution $\lambda$, then the boundary condition is satisfied and consequently

$$
\frac{\partial}{\partial \tau}\left(\frac{\partial u}{\partial \nu}+i \lambda u\right)=0 \text { on } \Gamma .
$$

Therefore in this case the derivative is given by

$$
\begin{aligned}
\frac{\partial G}{\partial z}(z, \lambda) h= & -k^{2} h_{\nu} u \circ z-\frac{\partial}{\partial \tau}\left(h_{\nu}\left(\frac{\partial u}{\partial \tau} \circ z\right)\right)+(i \lambda+H) h_{\nu} \frac{\partial u}{\partial \nu} \circ z \\
& +i h_{\nu}\left(\frac{\partial \lambda}{\partial \nu} u\right) \circ z .
\end{aligned}
$$


According to [6], where the work in [5] is extended to a non-constant impedance, this is up to a factor -1 the impedance boundary condition to the boundary value problem that characterizes the Fréchet derivative of the operator defined in (4). In other words and as expected, this is up to a factor -1 the boundary values of the extra forward problem that needs to be solved at each step of the Newton method applied to the operator defined in (4).

\section{The Hybrid Method}

We are interested in introducing the hybrid method as a numerical method to solve the inverse scattering problem for shape and impedance. In this section we will formulate the inverse problem in terms of $G$ and suggest the use of the results from the previous section to numerically solve it. However, the first issue that needs to be addressed is uniqueness of this inverse problem.

Theorem 4 (Uniqueness) The far-field patterns corresponding to an infinite number of plane waves with distinct directions uniquely determine the shape and location of the scatterer $D$ and the impedance function $\lambda$.

Prof. See [14, Thm. 2.1].

For numerical reconstructions using the hybrid method, we will consider as data the far field pattern for just one incident direction. Though there is to our knowledge no uniqueness result for this case, this makes sense by a formal argument. In fact, if one is given the far field pattern $u_{\infty}$ for one incident direction, which is a complex valued function on the unit circle or equivalently in $[0,2 \pi]$, one should formally be able to reconstruct two real valued functions on $[0,2 \pi]$. For instance, considering star-shaped domains, this two real functions could be the parameterization of the boundary $\Gamma$ of the scatterer and the impedance $\lambda$ defined on this boundary.

Keeping this in mind, in order to apply the theorems in the previous section on the differentiability of $G$, the impedance $\zeta$ must be defined at least in an open neighbourhood of the contour $\gamma$ parameterized by $z$. Therefore one needs to extend it into a neighbourhood since the impedance is only defined on the boundary by the boundary condition. On the one hand, one way to do it would be to extend its values along the normal direction to $\gamma$, which is possible in a neighbourhood of the contour since the contour is assumed to be $C^{2}$-smooth. Though this idea does not impose any restrictions on the domain, we would only be able to apply the method to continuously differentiable impedances, which is assuming more than we usually have in practice. On the other hand, assuming that the boundary of the domain is starlike, that is, it is parameterized by

$$
z(t)=\{r(t)(\cos t, \sin t): t \in[0,2 \pi]\}
$$

with some $2 \pi$ periodic positive $C^{2}$ function $r$, one could easily extend the impedance as a direction dependent function, that is, $\zeta=\zeta(\hat{x})$. In this way, the perturbations $h$ to the initial parameterization $z$ would also be starlike in the form of

$$
h(t)=\{q(t)(\cos t, \sin t): t \in[0,2 \pi]\}
$$


with some $2 \pi$ periodic $C^{2}$ function $q$ and therefore the last term on the right hand side of (35) would vanish since we would have

$$
\operatorname{grad} \zeta \cdot h=\frac{\partial \zeta}{\partial \theta} \theta \cdot h=0,
$$

where $\theta=\hat{x}^{\perp}$. Therefore we get

$$
\begin{aligned}
\frac{\partial}{\partial z} G(z, \zeta) h= & -k^{2} h_{\nu} u \circ z-\frac{\partial}{\partial \tau}\left(h_{\nu}\left(\frac{\partial u}{\partial \tau} \circ z\right)\right)+(i \zeta+H) h_{\nu} \frac{\partial u}{\partial \nu} \circ z \\
& +h_{\tau}\left[\left(\frac{\partial^{2} u}{\partial \tau \partial \nu}+i \zeta \frac{\partial u}{\partial \tau}\right) \circ z\right]
\end{aligned}
$$

which can be applied requiring the solution $\lambda$ just to be continuous, since no derivative of $\zeta$ is now required.

From this and also accordingly with the formal argument on "uniqueness", we will just consider star-shaped domains in the further analysis. With this assumption, for some range of impedances one can prove injectivity for the Fréchet derivative of $G$ if one is laying in the true solutions $\Gamma$ and $\lambda$ to the inverse problem.

Theorem 5 If the parametrization $z$ and the impedance $\lambda$ are the solutions of the inverse problem with $\lambda>k$ then

$$
\frac{\partial G}{\partial z}(z, \lambda) h+\frac{\partial G}{\partial \lambda}(z, \lambda) \mu=0
$$

implies that $h_{\nu}=0$ and $\mu=0$. In addition, for star-shaped domains, that is, if $z$ and hare of the form (39) and (40) respectively, then it also implies $h=0$.

Proof. We start by noting that if $z$ and $\lambda$ are correct then the boundary condition is satisfied and so (38) is valid. Therefore by assumption we have

$$
-k^{2} h_{\nu} u-\frac{\partial}{\partial \tau}\left(h_{\nu} \frac{\partial u}{\partial \tau}\right)+(i \lambda+H) h_{\nu} \frac{\partial u}{\partial \nu}+i h_{\nu}\left(\frac{\partial \lambda}{\partial \nu} u\right)+i \mu u=0
$$

Using the boundary condition again we get

$$
\left(k^{2}-\lambda^{2}\right) h_{\nu} u+\frac{\partial}{\partial \tau}\left(h_{\nu} \frac{\partial u}{\partial \tau}\right)+i\left(\lambda h_{\nu} H-h_{\nu} \frac{\partial \lambda}{\partial \nu}-\mu\right) u=0 \text { on } \Gamma
$$

We will now follow the ideas of Thm 2.2 in [14]. We start by noting that

$$
\frac{\partial}{\partial \tau}\left(h_{\nu} \frac{\partial u}{\partial \tau} \bar{u}\right)=\frac{\partial}{\partial \tau}\left(h_{\nu} \frac{\partial u}{\partial \tau}\right) \bar{u}+h_{\nu}\left|\frac{\partial u}{\partial \tau}\right|^{2}
$$

and as

$$
\frac{\partial|u|^{2}}{\partial \tau}=2 \operatorname{Re}\left(\frac{\partial u}{\partial \tau} \bar{u}\right)
$$

one gets

$$
\operatorname{Re}\left(\frac{\partial}{\partial \tau}\left(h_{\nu} \frac{\partial u}{\partial \tau}\right) \bar{u}\right)=\frac{1}{2} \frac{\partial}{\partial \tau}\left(h_{\nu} \frac{\partial|u|^{2}}{\partial \tau}\right)-h_{\nu}\left|\frac{\partial u}{\partial \tau}\right|^{2}
$$


Now multiplying (42) by $\bar{u}$ and taking the real part we get

$$
\left(k^{2}-\lambda^{2}\right) h_{\nu}|u|^{2}+\frac{1}{2} \frac{\partial}{\partial \tau}\left(h_{\nu} \frac{\partial|u|^{2}}{\partial \tau}\right)-h_{\nu}\left|\frac{\partial u}{\partial \tau}\right|^{2} \text { on } \Gamma
$$

We now assume that $h_{\nu}$ does not identically vanish. One can assume without loss of generality that the set $\Sigma=\left\{x \in \Gamma: h_{\nu}>0\right\}$ is nonempty and integrating over this set one gets

$$
\int_{\Sigma}\left[\left(k^{2}-\lambda^{2}\right)|u|^{2}-\left|\frac{\partial u}{\partial \tau}\right|^{2}\right] h_{\nu} d s=0 .
$$

If $\lambda>k$ then this implies that $u=0$ on $\Sigma$ and by the boundary condition the normal derivative of $u$ in this set also vanishes. Therefore by Holmgreen's theorem this implies that the total field $u$ vanishes in $\mathbb{R}^{2} \backslash \bar{D}$ which is a contradiction. Then we conclude that $h_{\nu}=0$ on $\Gamma$. By (42) this implies that $u \mu=0$, which by a similar argument as before implies $\mu=0$.

Using the extra assumptions (39) and (40) on $z$ and $h$ respectively, one can conclude that

$$
h_{\nu}=\frac{r q}{\sqrt{r^{2}+r^{\prime 2}}}
$$

and so $h_{\nu}=0$ implies that $h=0$.

We are now in a position to present the hybrid method. As already mentioned it combines ideas of both iterative and decomposition methods. As in the latter, it consists of two steps. In the first step, one deals with the ill-posedness in the spirit of the potential method of Kirsch and Kress $[8,9,10]$. Given an approximation $\gamma$ with parameterization $z$ of the form (39), we start by solving the far field equation

$$
F_{\infty, \gamma} \varphi=u_{\infty}
$$

with respect to $\varphi$. The operator on the left hand side is injective, however, as it is a compact operator, its inversion is ill-posed and therefore stabilization is needed. For this, we suggest using the well established Tikhonov regularization. Settling the first step, with an approximate solution $\varphi$ to (43) we then obtain an approximation to the total field by setting

$$
u=u^{i}+\left(K_{\gamma}-i \eta S_{\gamma}\right) \varphi \quad \text { in } \mathbb{R}^{2} \backslash \gamma .
$$

We now recall the parameterization to the boundary condition operator $G$ introduced in (14). In order to satisfy the boundary condition, we need to find an updated parameterization $z+h$ and an updated impedance $\zeta+\mu$ such that

$$
G(z+h, \zeta+\mu)=0 .
$$

Therefore, in a second step, as in the classical Newton method, we solve the linearized equation

$$
G(z, \zeta)+\frac{\partial G}{\partial z}(z, \zeta) h+\frac{\partial G}{\partial \zeta}(z, \zeta) \mu=0
$$

with respect to $h$ and $\mu$ in a least squares sense. Our hybrid method then consists in repeating both steps iteratively until some stopping criteria is fulfilled.

We point out that this method does not need a forward solver at each iteration step which reduces the computational costs. As we will see in section 6 , this does not deteriorate the 
reconstructions. Therefore the method combines the advantages of both Newton type and decomposition methods.

Remark 3 Note that the approximation of the total field $u$ given by (44) has a jump on $\gamma$. Therefore, at each collocation point considered for solving (45) in a least squares sense a choice has to be made whether to use the interior or exterior values for $u$ to compute the Fréchet derivative of $G$ given in Theorem 3. Here we assume as a priori knowledge that the initial guess $\gamma$ lies inside the scatterer $D$ and consider the exterior field for computations.

\section{An Optimization Problem}

Along the lines of section 5.4 in [4], we will now relate the hybrid method with a minimization problem as its theoretical background. The main difference is that in [4] the analysis is done for a single layer representation of the solution and therefore the compactness of the single and the double layer operators is a key ingredient for the results obtained. In this case, the operator $T$ ruins this compactness. To avoid this problem one assumes more regularity on the density $\varphi$.

We will stick to the restriction to star-shaped domains, that is,

$$
\gamma_{r}=\{z(t)=r(t)(\cos t \sin t) \mid r \text { is } 2 \pi \text {-periodic }\} .
$$

and consider only functions $r \in U$, where

$$
U=\left\{r \in H^{l}[0,2 \pi] \mid 0<r_{i} \leq r(t) \leq r_{e} t \in[0,2 \pi]\right\}
$$

for some fixed $r_{i}, r_{e} \in \mathbb{R}^{+}$. We will also consider impedances $\zeta \in V$ given by

$$
V=\left\{\zeta: \gamma \rightarrow \mathbb{R} \mid \zeta \in H^{p}(\gamma), 0 \leq \zeta(x) \leq \zeta_{e} \forall x \in \gamma\right\}
$$

for some fixed $\zeta_{e} \in \mathbb{R}^{+}$. For densities space we will consider $\varphi \in H^{q}(\gamma)$.

Assuming $q>3 / 2+\alpha$, for some fixed $\alpha>0$, we can restrict the operators $F_{\infty, \gamma}$ and $B_{\gamma, \zeta}$ introduced in section 2 as operators mapping from $H^{q}(\gamma)$ to $L^{2}(\Omega)$ and $L^{2}(\gamma)$, respectively.

We define the cost function $\Lambda(r, \zeta, \varphi ;):. U \times V \times H^{q}(\gamma) \rightarrow \mathbb{R}_{0}^{+}$given by

$$
\Lambda(r, \zeta, \varphi ; \beta)=\beta\|\varphi\|_{H^{q}\left(\gamma_{r}\right)}^{2}+\Lambda_{1}(r, \zeta, \varphi)+\Lambda_{2}(r, \zeta, \varphi)
$$

for $\beta>0$, where

$$
\Lambda_{1}(r, \zeta, \varphi)=\left\|F_{\infty, \gamma_{r}} \varphi-f\right\|_{L^{2}(\Omega)}^{2}
$$

for some $f \in L^{2}(\Omega)$ and

$$
\Lambda_{2}(r, \zeta, \varphi)=\left\|B_{\gamma_{r}, \zeta} \varphi+g\right\|_{L^{2}\left(\gamma_{r}\right)}^{2}
$$

for some $g \in L^{2}\left(\mathbb{R}^{2}\right)$, where $\gamma_{r}$ is the contour corresponding to $r$.

On the one hand, if $f=u_{\infty}$ and if $r$ and $\zeta$ are fixed, the minimization of

$$
\beta\|\varphi\|_{H^{q}\left(\gamma_{r}\right)}^{2}+\Lambda_{1}(r, \zeta, \varphi)
$$

with respect to $\varphi$ is equivalent to finding a regularized solution to (43) by Tikhonov regularization with parameter $\beta$. On the other hand, if $g$ is the impedance boundary data 
and $\varphi$ is kept fixed, minimizing $\Lambda_{2}(r, \zeta, \varphi)$ in terms of $r$ and $\zeta$ can be seen as finding a solution to (45). In this way this nonlinear optimization problem is related with the inverse scattering problem.

Given $f$ and $g$, we define the pair $\left(r_{0}, \zeta_{0}\right) \in U \times V$ as being optimal if there exists $\varphi_{0} \in H^{q}\left(\gamma_{0}\right)$ such that

$$
\Lambda\left(r_{0}, \zeta_{0}, \varphi_{0} ; \beta\right)=m(\beta)
$$

where

$$
m(\beta):=\inf _{r \in U, \zeta \in V, \varphi \in H^{q}(\gamma)} \Lambda(r, \zeta, \varphi ; \beta) .
$$

We will now present some results on this optimization problem.

Theorem 6 Assume in addition to $q>3 / 2+\alpha$ that also $l>5 / 2$ and $p>1 / 2$. Then for each $\beta>0, f \in L^{2}(\Omega)$ and $g \in L^{2}\left(\mathbb{R}^{2}\right)$ there exists an optimal pair $(r, \zeta) \in U \times V$.

Proof. Assume the triple $\left(r_{n}, \zeta_{n}, \varphi_{n}\right)$ to be a minimizing sequence, that is,

$$
\lim _{n \rightarrow \infty} \Lambda\left(r_{n}, \zeta_{n}, \varphi_{n} ; \beta\right)=m(\beta) .
$$

As $U$ is bounded, by the Sobolev compact embedding $H^{l}[0,2 \pi] \subset C^{2}[0,2 \pi]$ one has that $U$ is compact in $C^{2}[0,2 \pi]$. Therefore, without loss of generality, we can assume $C^{2}$-convergence $r_{n} \rightarrow r$ as $n \rightarrow \infty$. As $\mathrm{U}$ is closed, $r \in U$. In a similar way one can assume $C$-convergence of $\zeta_{n} \rightarrow \zeta \in V$.

One also has that

$$
\beta\left\|\varphi_{n}\right\|_{H^{q}\left(\gamma_{r}\right)}^{2} \leq \Lambda\left(r_{n}, \zeta_{n}, \varphi_{n} ; \beta\right) \rightarrow m(\beta)
$$

as $n \rightarrow \infty$, so $\varphi_{n}$ is bounded and by a similar argument using the compact embedding $H^{l}[0,2 \pi] \subset C^{1, \alpha}[0,2 \pi]$ one can assume that $\varphi_{n} \rightarrow \varphi$. By continuity of the functional $\Lambda$ in all its variables, one has the result, since

$$
\Lambda(r, \zeta, \varphi ; \beta)=\lim _{n \rightarrow \infty} \Lambda\left(r_{n}, \zeta_{n}, \varphi_{n} ; \beta\right)=m(\beta) \text {. }
$$

We are interested in the behaviour of a solution to the minimization problem as the regularization parameter goes to zero. One can state the following convergence result. Note that, unfortunately, it does not imply convergence to a solution of the inverse scattering problem.

Theorem 7 Assume $q>3 / 2+\alpha, l>5 / 2$ and $p>1 / 2$. Assume also that $f$ is the exact far-field pattern $u_{\infty}$, that $g$ is the exact boundary data $g=\partial u^{i} / \partial \nu+i \lambda u^{i}$, that the solution $\Gamma$ can be parameterized by some $r_{\Gamma} \in U$ and that the exact impedance $\lambda \in V$. Let $\left(\beta_{n}\right)$ be a null sequence and let $\left(r_{n}, \zeta_{n}\right)$ be the sequence of corresponding optimal pairs. Then there exists a convergent subsequence of $\left(r_{n}, \zeta_{n}\right)$ and every limit point $\left(r^{*}, \zeta^{*}\right)$ represents a curve and impedance such that

$$
\frac{\partial u^{s}}{\partial \nu}+i \zeta^{*} u^{s}=-g \text { on } \gamma^{*}
$$


Proof. From section 2, one knows that the solution to the direct problem can be represented by a combined single and double layer potential via the solution $\varphi$ of (10). Therefore with the assumptions on $f$ and $g$ one has that

$$
\Lambda_{1}\left(r_{\Gamma}, \lambda, \varphi\right)=\Lambda_{2}\left(r_{\Gamma}, \lambda, \varphi\right)=0 .
$$

Then

$$
\lim _{\beta \rightarrow 0} m(\beta)=0
$$

since

$$
m(\beta) \leq \Lambda\left(r_{\Gamma}, \lambda, \varphi ; \beta\right)=\beta\|\varphi\|_{H^{q}\left(\gamma_{r}\right)}^{2} .
$$

The existence of a convergent subsequence $\left(r_{k(n)}, \zeta_{k(n)}\right)_{n \in \mathbb{N}}$ comes from the proof of theorem 6. For simplicity we will denote $k=k(n)$. Let $\left(r^{*}, \zeta^{*}\right)$ be the limit point of that convergent subsequence and let $u^{*}$ be the solution to the direct scattering problem with boundary condition

$$
\frac{\partial u^{*}}{\partial \nu}+i \zeta^{*} u^{*}=-g \quad \text { on } \gamma^{*}
$$

Since $\left(r_{k}, \zeta_{k}\right)$ is optimal there exists $\left(\varphi_{k}\right)_{k \in \mathbb{N}}$ such that

$$
\Lambda\left(r_{k}, \zeta_{k}, \varphi_{k}, \beta_{k}\right)=m\left(\beta_{k}\right) .
$$

Let now $u_{k}$ be the combined single and double layer potential over $\gamma_{k}$ applied to the density $\varphi_{k}$. The potential $u_{k}$ can be interpreted as the solution to the exterior scattering problem with boundary $\gamma_{k}$ and impedance $\zeta_{k}$.

From (46),

$$
\left\|\frac{\partial u_{k}}{\partial \nu}+i \zeta_{k} u_{k}+g\right\|_{L^{2}\left(\gamma_{k}\right)}^{2} \leq m\left(\beta_{k}\right) \rightarrow 0
$$

and

$$
\left\|F_{\infty, \gamma_{k}} \varphi_{k}-f\right\|_{L^{2}(\Omega)}^{2} \leq m\left(\beta_{k}\right) \rightarrow 0
$$

both as $k \rightarrow \infty$.

By (47) one concludes that $u_{k}$ and all its derivatives converge to $u^{*}$ on compact sets of the exterior domain (e.g. thm. 5.16 in [4]) and so the far field patterns of $u_{k}$ also converge to the far field pattern $u_{\infty}^{*}$ of $u^{*}$. By (48) we conclude that $u_{\infty}^{*}=f=u_{\infty}$ and so $u^{s}=u^{*}$ follows. This concludes the proof.

\section{Numerical Results}

In this final section we describe some details on the numerical implementation of the method.

For all the examples presented, we fixed the wave number $k=1$ and used as incident field

$$
u^{i}(x)=e^{i k x . d}
$$


that is, a plane wave with direction $d \in \Omega$.

The synthetic far field data was obtained through the process described in section 2 . We computed the far field pattern for one incident direction at 60 equidistant points on the unit circle $\Omega$ and considered it as the given data for the inverse scattering problem.

For the first step of the method, equation (43) was solved by Tikhonov regularization, considering 40 points over the boundary $\gamma$. For regularization parameter we used $\beta=0.5^{n} \times 10^{-10}$, where $n$ is the number of iterations.

For each iteration, in the second step of the method both the function $u$ and its normal derivative $\partial u / \partial \nu$ have to be computed. This was done evaluating their representation integral formula considered in section 2 using the trigonometric quadrature rules described in [12] and in [4, Ch. 3.5]. The coupling parameter for the combined single and double layer potential was chosen as $\eta=k$, as suggested in [11]. For the tangential derivatives occurring in the expressions for $G^{\prime}$ we used trigonometric differentiation, that is, we interpolated by a trigonometric polynomial and took its derivative as approximation.

As parameterization space for the radius function we considered trigonometric polynomials

$$
r(t)=\sum_{j=0}^{N_{p}} a_{j}^{(p)} \cos j t+\sum_{j=1}^{N_{p}} b_{j}^{(p)} \sin j t
$$

of degree $N_{p}=5$. For impedance space we have also used trigonometric polynomials

$$
\lambda(t)=\sum_{j=0}^{N_{l}} a_{j}^{(l)} \cos j t+\sum_{j=1}^{N_{l}} b_{j}^{(l)} \sin j t
$$

with $N_{l}=4$. Numerical experiments showed us that better results were obtained when $N_{p} \geq N_{l}$.

As stopping criteria as residual associated with each iteration step the sum of the cost functions $\Lambda_{1}(r, \zeta, \varphi)+\Lambda_{2}(r, \zeta, \varphi)$ defined in the previous section in the following way. We computed this residual for the current approximation. Then we solved (45) to get the candidate for a new approximation by a Levenberg-Marquardt step to improve on the stability of the method. As regularization parameter for the Levenberg-Marquardt step we started with $10^{-5}$ and if the residual for the new approximation would be larger than for the current approximation, we would increase the Levenberg-Marquardt parameter by a factor of 10 and repeat the second step. Otherwise we would take the new approximation and proceed with the next iteration repeating both steps of the method. The method was stopped when the regularization parameter for the Levenberg-Marquardt step became equal to $10^{-1}$. In the examples presented, the stopping criteria was achieved after about 10 iterations.

We will show two examples of numerical reconstructions. As a first example we considered the domain parameterized by

$$
z^{(1)}(t)=(2+0.3 \cos 3 t)\{\cos (t), \sin (t)\}, \quad t \in[0,2 \pi],
$$

the impedance

$$
\lambda^{(1)}(t)=1.5-\cos t+0.5 \sin 2 t, \quad t \in[0,2 \pi]
$$


and the incident direction $d^{(1)}=(-1,0)$.

As a second example we used the peanut shaped domain parameterized by

$$
z^{(2)}(t)=3 \sqrt{\cos ^{2} t+0.25 \sin ^{2} t}\{\cos (t), \sin (t)\}, \quad t \in[0,2 \pi],
$$

the impedance

$$
\lambda^{(2)}(t)=1+\sin ^{3} t, \quad t \in[0,2 \pi]
$$

and the incident direction $d^{(2)}=(0,1)$.

The reconstructions obtained can be seen in figures $1-4$. We represent in grey the solution, in black the approximation and the dashed line is the initial guess. For both examples we used as initial guess a circle of radius 1.5 and a constant impedance $\lambda=1.5$.
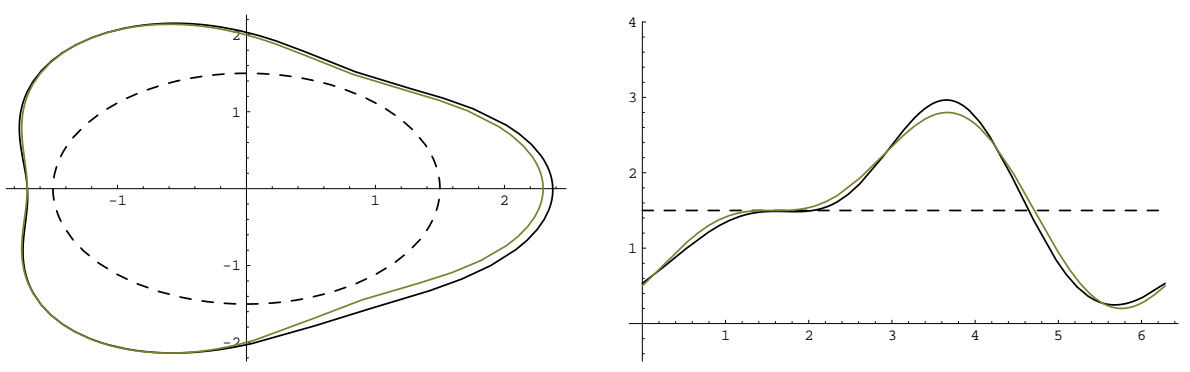

Figure 1. Reconstruction without noise for the first example.
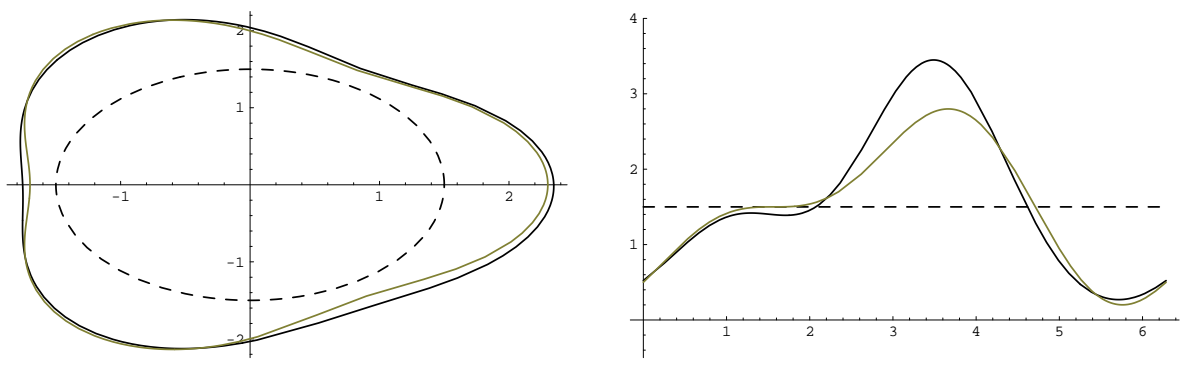

Figure 2. Reconstruction with $2 \%$ noise for the first example.
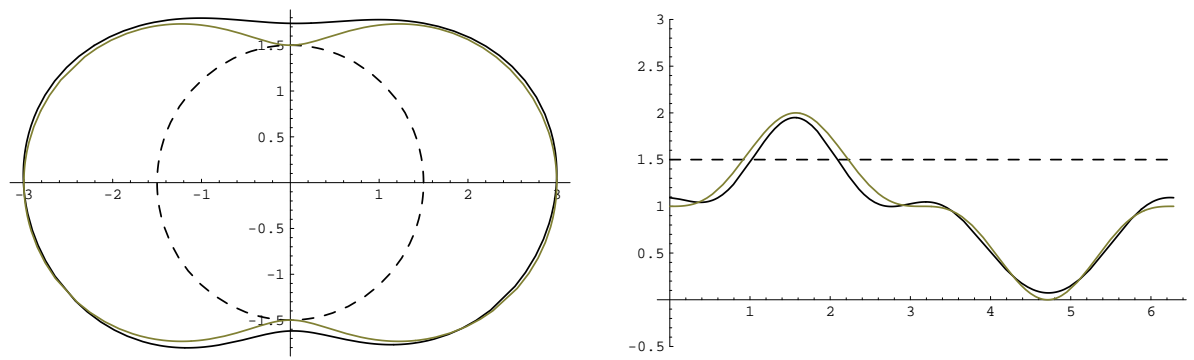

Figure 3. Reconstruction without noise for the second example.

It is clear that the obstacle is usually better reconstructed than the impedance, in accordance with [14]. In comparison with [14], our reconstructions do not deteriorate if the 

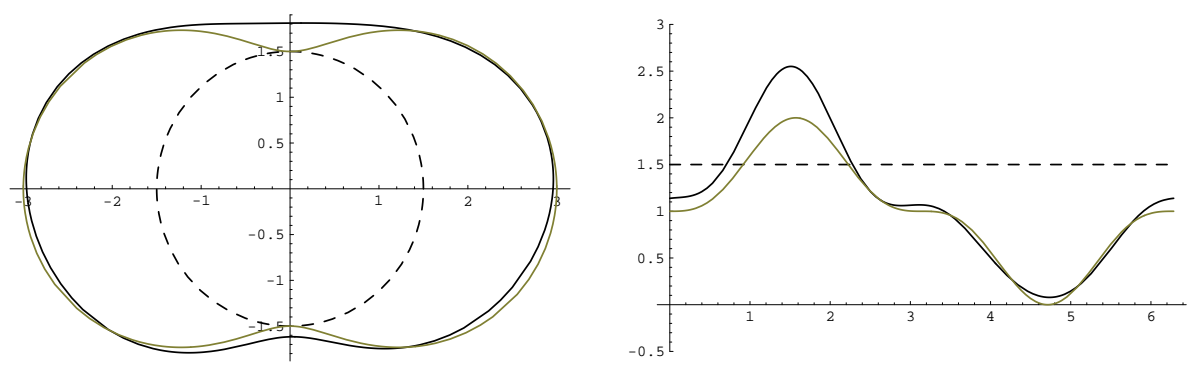

Figure 4. Reconstruction with $2 \%$ noise for the second example.

amplitude of the impedance is increased nor if the impedance is close to zero, especially if these features occur in the illuminated area of the scatterer. However, problems might occur when recovering maximums and minimums of the impedance in the shadow region, as shown in the reconstructions with noise. As expected, better reconstructions were obtained when the illuminated area was greater or equal to the shadow one.

\section{Acknowledgments}

The author would like to thank Prof. Dr. Rainer Kress for the helpful discussions and suggestions on the topic of this work. The support of the author's work by Fundacão para a Ciência e a Tecnologia (Portuguese Foundation for Science and Technology) through the scholarship SFRH/BD/14248/2003 is gratefully acknowledged.

\section{References}

[1] Cakoni F., Colton D., The determination of the surface impedance of a partially coated obstacle from far-field data, SIAM J. Appl. Math., Vol.64,No.2, pp. 709-723, 2004

[2] Chapko R., Kress R., A hybrid method for inverse boundary values problems in potential theory, J. Inverse Ill-Posed Problems 13, 1-14, 2005

[3] Colton D., Kirsch A., A simple mtehod for solving inverse scattering problems in the resonace region, Inverse Problems 12, pp.383-393

[4] Colton D., Kress R., Inverse Acoustic and Electromagnetic Scattering Theory, $2^{\text {nd }}$ Edition, Springer, 1998

[5] Hettlich F., Fréchet derivatives in inverse obstacle scattering, Inverse Problems 11, 371-382, 1995, (erratum, Inverse Problems 14, 204-210, 1998)

[6] Hettlich F., The Domain derivative in Inverse Obstacle Problems, Habilitationsschrift, 1999

[7] Hohage T., Convergence rates of a regularized Newton method in sound-hard inverse scattering, SIAM J. Numer. Anal. 36, 125-142, 1998

[8] Kirsch A., Kress R., On an integral equation of the first kind in inverse acoustic scattering, Inverse Problems (Cannon and Hornung, eds.), ISNM 77, 93-102, 1986

[9] Kirsch A., Kress R., A numerical method for an inverse scattering problem, Inverse Problems (Engl and Groetsch, eds.), Academic Press, Orlando, 279-290, 1987

[10] Kirsch A., Kress R., An optimization method in inverse acoustic scattering, Boundary Elements IX, Vol.3 Fluid Flow and Potential Applications (Brebbia et al., eds), Springer Verlag, Berlin Heidelberg New York, 3-18, 1987

[11] Kress R., Minimizing the condition number of boundary integral operators in acoustic and electromagnetic scattering, Quart. J. Mech. Appl. Math. 38,323-341, 1985 
[12] Kress R., On the numerical solution of a hypersingular integral equation in scattering theory, J. Comp. Appl. Math. 61,345-360, 1995

[13] Kress R., Newton's Method for inverse obstacle scattering meets the method of least squares, Inverse Problems 19, 91-104, 2003

[14] Kress R., Rundell W., Inverse Scattering for shape and impedance, Inverse Problems 17, 1075-1085, 2001

[15] Kress R., Serranho P., A hybrid method for two-dimensional crack reconstruction, Inverse Problems 21,773-784, 2005

[16] Kress R., Serranho P., A hybrid method for sound-hard obstacle reconstruction, (to appear)

[17] Potthast R., Fréchet differentiability of the solution to the acoustic Neumann scattering problem with respect to the domain, J. Inverse Ill-posed Problems 4, 67-84, 1996

[18] Potthast R., On the convergence of a new Newton-type method in inverse scattering, Inverse Problems 17, 1419-1434, 2001

[19] Roger A., Newton Kantorovich algorithm applied to an electromagnetic inverse problem, IEEE Trans. Ant. Prop. AP-29, 232-238, 1981. 